\title{
Selenium-Containing Heterocycles From Isoselenocyanates: Synthesis of Ethyl 4-Oxo-2-amino-4,5-dihydroselenophene-3-carboxylates
}

\author{
Sommen, G L ; Linden, Anthony ; Heimgartner, H
}

\begin{abstract}
Aryl and alkyl isoselenocyanates 2 react with ethyl -chloroacetoacetate (1) in the presence of triethylamine to give the corresponding 4-oxo-2-amino-4,5-dihydroselenophene-3-carboxylates $4 \mathrm{a}-\mathrm{f}$ in moderate to good yields.
\end{abstract}

DOI: https://doi.org/10.2174/157017807780037342

Posted at the Zurich Open Repository and Archive, University of Zurich

ZORA URL: https://doi.org/10.5167/uzh-50685

Journal Article

Accepted Version

Originally published at:

Sommen, G L; Linden, Anthony; Heimgartner, H (2007). Selenium-Containing Heterocycles From Isoselenocyanates: Synthesis of Ethyl 4-Oxo-2-amino-4,5-dihydroselenophene-3-carboxylates. Letters in Organic Chemistry, 4(1):7-12.

DOI: https://doi.org/10.2174/157017807780037342 


\title{
Selenium-Containing Heterocycles From Isoselenocyanates: Synthesis of Ethyl 4-Oxo-2-amino-4,5-dihydroselenophene-3-carboxylates
}

\author{
Geoffroy L. Sommen, ${ }^{1)}$ Anthony Linden, Heinz Heimgartner* \\ Institute of Organic Chemistry, University of Zürich, Winterthurerstrasse 190, CH-8057 Zürich, \\ Switzerland
}

\begin{abstract}
Aryl and alkyl isoselenocyanates 2 react with ethyl $\gamma$-chloroacetoacetate (1) in the presence of triethylamine to give the corresponding 4-oxo-2-amino-4,5dihydroselenophene-3-carboxylates 4a-f in moderate to good yields.
\end{abstract}

Key Words: Selenium heterocycles, Isoselenocyanates, Selenophenes, Cyclization, $\beta$ Ketoester, Crystal structure

\section{INTRODUCTION}

Selenium containing heterocycles are of increasing interest because of the antitumor, antibacterial and other biological activities of some representatives [1]. During our efforts devoted to the chemistry of selenaheterocycles, we also became interested in the synthesis of selenophenes. There are many articles and reviews dealing with the preparation of selenophenes [2], however, to the best of our knowledge, no synthesis starting from isoselenocyanates has been published. On the other hand, several procedures concerning the preparation of thiophenes by using isothiocyanates have been described [3]. For example, syntheses of some 4-oxothiophenes have been reported by Faull and Hull [4] as well as by Ibrahim, Sadek, Aziz, and Elnagdi [5] in the 80's. Compounds of this type, including 4-oxofurans, are known as novel antiallergic agents [6].

\footnotetext{
* Address correspondence to this author at the Institute of Organic Chemistry, University of Zürich, Winterthurerstrasse 190, CH-8057 Zürich, Switzerland; E-mail: heimgart@oci.unizh.ch; ${ }^{1)}$ Present address: Lonza AG, Walliser Werke, CH-3930, Visp, Switzerland; e-mail: geoffroy.sommen@lonza.com
} 
As a part of our program aimed at the development of simple procedures for the preparation of selenium-containing heterocycles [7-14], we have recently reported on the utility of isoselenocyanates for the synthesis of selenazetidines [15], selenazolidines [16,17], selenazines [16,18], selenadiazines [19], selenazepines [20], selenorhodanines [21], and triazoleselones [22]. As a continuation of this work, we report here on a novel and efficient synthesis of selenophene derivatives.

It was expected that the carbanion generated from ethyl $\gamma$-chloroacetoacetate (1) and triethylamine in DMF reacts with aromatic and aliphatic isoselenocyanates $\mathbf{2}$ to give intermediate keten-N,Se-acetals of type 3. The latter, in an intramolecular nucleophilic substitution, can cyclize to give the selenophene ring system 4 (Scheme 1). Alternatively, ring closure via nucleophilic substitution by the $\mathrm{N}$-atom could lead to pyrrolidine derivatives of type 5. Analogous ring closure reactions, which gave azoleselones, have been described earlier [21,22]. The necessary isoselenocyanates $\mathbf{2}$ can be prepared easily from the corresponding $\mathrm{N}$-arylformamides by treatment with phosgene and elemental selenium by following a slightly modified Barton procedure [23].

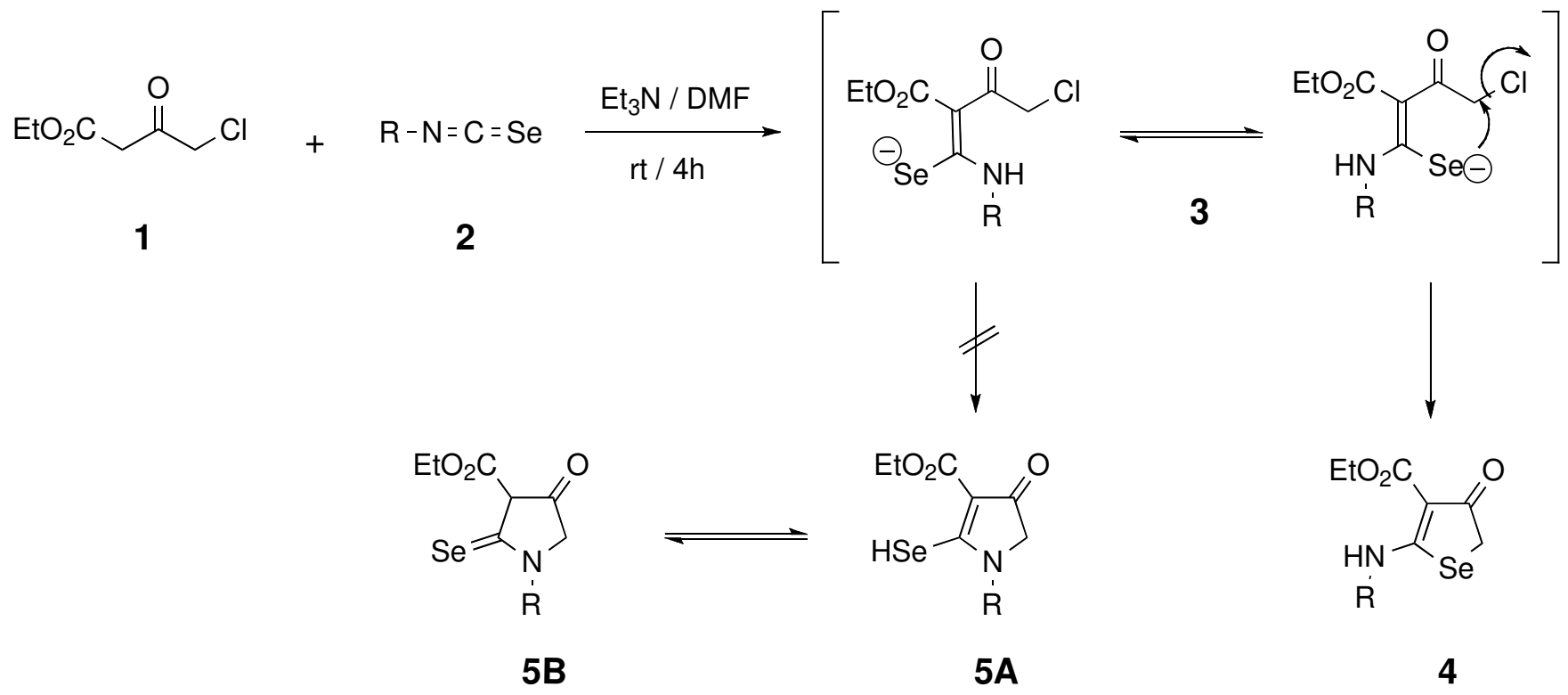

Scheme 1

\section{RESULTS AND DISCUSSION}

After stirring an equimolar mixture of ethyl $\gamma$-chloroacetoacetate (1), the respective isoselenocyanate 2, and triethylamine in DMF at room temperature for four hours, the solvent was 
evaporated. Column chromatography of the solid residue on silica gel with hexane/ethyl acetate and recrystallization of the major product from ethyl acetate gave ethyl 2-amino-4,5-dihydro-4oxoselenophene-3-carboxylates 4 in fair yields (Table 1).

Table 1: Preparation of Selenophenes 4 from Isoselenocyanates 2

Entry


The structures of the products were established on the basis of their spectroscopic data and elemental analyses. Indicative are the IR absorptions at ca. 1660 and $1640 \mathrm{~cm}^{-1}$ for the keto and ester $\mathrm{C}=\mathrm{O}$ groups, respectively. In the mass spectra (CI mode), the respective $[\mathrm{M}+1]^{+}$peak appears as the base peak (100\%), and the ${ }^{1} \mathrm{H}$ NMR spectra show the expected signals. Most informative are the ${ }^{13} \mathrm{C}$ NMR spectra with three signals for $\mathrm{sp}^{2}-\mathrm{C}$ atoms at ca. 193, 182, and $167 \mathrm{ppm}$ for $\mathrm{C}(4)=\mathrm{O}, \mathrm{C}(2)$, and $\mathrm{CO}_{2} \mathrm{Et}$, respectively. The atom $\mathrm{C}(3)$ absorbs at ca. $99 \mathrm{ppm}$. In the case of $\mathbf{4 b}$, an X-ray crystal-structure determination was carried out, which confirmed the proposed structure (Fig. 1).

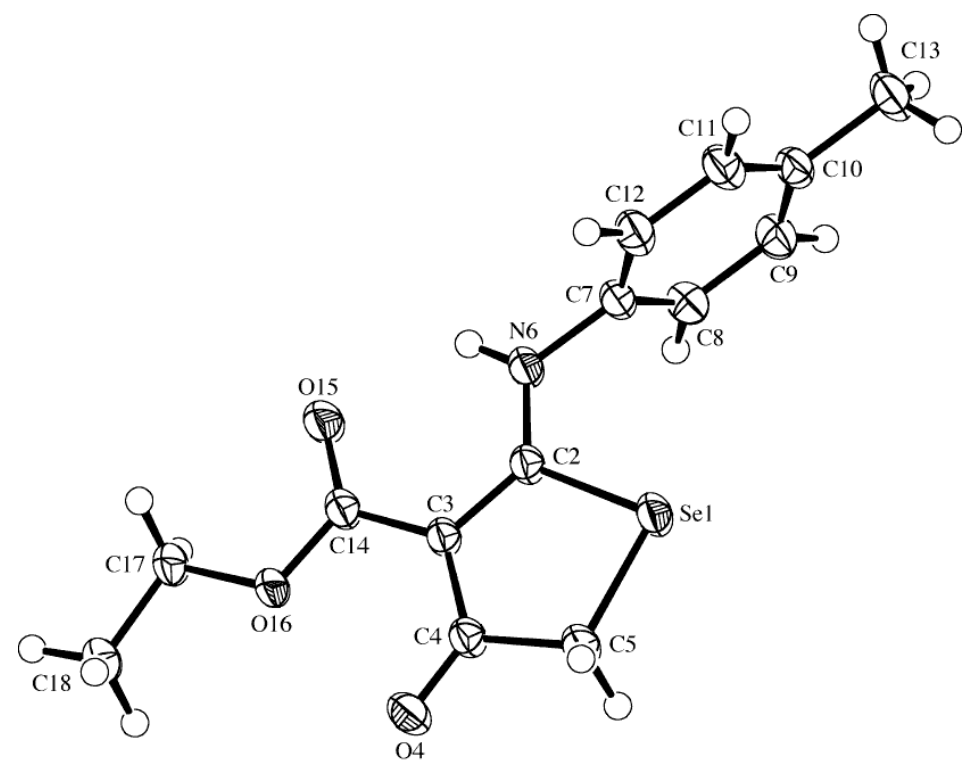

Fig. (1). ORTEP plot [24] of the molecular structure of $\mathbf{4 b}$ (50\% probability ellipsoids; arbitrary numbering of atoms)

In the crystal structure of $\mathbf{4 b}$ all atoms of the molecule, except for the atoms of the 4-methylphenyl ring, form a planar system. When calculated using all atoms except $C(8)$ to $C(13)$, the maximum deviation from the plane is $0.054(2) \AA$ for $\mathrm{C}(17)$. The plane of the 4-methylphenyl ring lies perpendicular to the heterocyclic ring plane [angle: $\left.88.94(7)^{\circ}\right)$ ]. The amine group $H N(6)$ forms bifurcated hydrogen bonds. One interaction is intramolecular with the ester carbonyl $\mathrm{O}(15)$ atom, while the second interaction is intermolecular with the same $\mathrm{O}$-atom of a neighboring molecule, thereby linking pairs of molecules about a centre of inversion into dimeric moieties. The intramolecular interaction forms a closed loop with the graph set motif [25] of $S(6)$, while the intermolecular interaction 
creates a loop with the graph set motif of $\mathrm{R}^{2}{ }_{2}(12)$. Taking both interactions together generates a fourmembered loop with a binary graph set motif of $\mathrm{R}^{2}{ }_{2}(4)$.

In summary, we have evidenced that ethyl $\gamma$-chloroacetoacetate $(\mathbf{1})$ is a very good precursor of selenophenes 4. The base-catalyzed reaction with isoselenocyanates 2 proceeds via addition of the carbanion of $\mathbf{1}$ onto the isoselenocyanate to yield the acyclic intermediate $\mathbf{3}$, which then undergoes a cyclization via the selenium atom to give 4 via elimination of $\mathrm{HCl}$. The alternative ring closure via nucleophilic attack of the nitrogen atom of $\mathbf{3}$ (cf. [21,22]), which would lead to a pyrrole derivative, was not observed as a result of the higher nucleophilicity of the selenium atom. Thus, the selenophenes 4 have been easily prepared in a one-pot procedure starting from aryl and cyclohexyl isoselenocyanates. These building blocks are less toxic, relatively stable, safe and easy to handle, and can be synthesized conveniently.

\section{EXPERIMENTAL}

\section{General}

TLC: silica gel $60 \mathrm{~F}_{254}$ plates $(0.25 \mathrm{~mm}$; Merck). Column chromatography (CC): silica gel 60 (0.040-0.063 mm; Merck). Melting Points: Büchi B-540 apparatus, in capillaries; uncorrected. ${ }^{1} \mathrm{H}-\mathrm{NMR}$ (300 MHz) and ${ }^{13} \mathrm{C}-\mathrm{NMR}(75.5 \mathrm{MHz})$ spectra: Bruker ARX-300 instrument in $\mathrm{CDCl}_{3}$; chemical shifts in ppm. CI-MS: Finnigan SSQ-700 or MAT-90 instrument; $\mathrm{NH}_{3}$ as carrier gas.

\section{Starting materials}

Malononitrile and all halogenated compounds are commercially available (Fluka). Isoselenocyanates were prepared according to Barton's procedure [23] by starting from formamides. Formanilide and $\mathrm{N}$-cyclohexylformamide are commercial products (Fluka and Aldrich), $\mathrm{N}$-(4methylphenyl)-, $N$-(4-bromophenyl)-, $N$-(2,6-dimethylphenyl)- and $N$-(4-methoxyphenyl)formamide were prepared from the respective aniline and 95\% formic acid [26]. The solution was heated to reflux for $30 \mathrm{~min}$ and evaporated to dryness in vacuo. The residue was dissolved in ether and washed with diluted acetic acid (5\%), water, and aqueous $\mathrm{NaHCO}_{3}$ (5\% aq.). The aqueous layer was extracted with ether, the combined organic extracts were dried with magnesium sulphate and evaporated under reduced pressure. The crude products were purified by recrystallization in EtOH/water.

\section{General Procedure for the Preparation of 4,5-Dihydroselenophene-3-carboxylates 4a-f}


A $25 \mathrm{~mL}$ round-bottom flask equipped with magnetic stirrer and condenser was charged with a solution of ethyl $\gamma$-chloroacetoacetate $(\mathbf{1}, 0.14 \mathrm{~mL}, 1.03 \mathrm{mmol})$ in DMF $(20 \mathrm{~mL})$. Triethylamine $(0.14$ $\mathrm{mL}, 1.03 \mathrm{mmol}$ ) was added and the mixture was stirred for $45 \mathrm{~min}$ at room temperature. Isoselenocyanate $(2,1.03 \mathrm{mmol})$ was added and the mixture was stirred for $4 \mathrm{~h}$ at room temperature before being evaporated to dryness under reduced pressure. The crude product was purified by column chromatography on silica gel without any further treatments by using hexane/ethyl acetate (100/0 to 50/50) as the eluant and crystallized from ethyl acetate.

\section{Ethyl 4,5-dihydro-4-oxo-2-(phenylamino)selenophene-3-carboxylate (4a)}

Yield: $52 \%$. White crystals. M.p. $152-154^{\circ} \mathrm{C}$. IR (KBr): $3441 w$ br, 3052w, 2983w, 1645s, 1588w, $1550 s, 1498 w, 1471 w, 1410 m, 1381 m, 1351 w, 1282 w, 1219 m, 1036 m, 946 w, 780 w, 736 w .{ }^{1} \mathrm{H}$ NMR $\left(\mathrm{CDCl}_{3}\right): 1.65$ (t, J=7.1 Hz, 3H, $\left.\mathrm{CH}_{3}\right) ; 3.96\left(\mathrm{~s}, 2 \mathrm{H}, \mathrm{CH}_{2}\right) ; 4.61$ (q, J=7.1 Hz, 2H, $\mathrm{CH}_{2} \mathrm{O}$ ); 7.62-7.73 (m, 5H, arom. $\mathrm{CH}) .{ }^{13} \mathrm{C} \mathrm{NMR}\left(\mathrm{CDCl}_{3}\right): 14.3\left(\mathrm{CH}_{3}\right) ; 32.6\left(\mathrm{CH}_{2}\right) ; 60.4\left(\mathrm{CH}_{2} \mathrm{O}\right) ; 99.8(\mathrm{C}(3)) ; 124.1$ (2 arom. $\mathrm{CH}) ; 128.0$ (1 arom. CH); 129.7 (2 arom. $\mathrm{CH}) ; 138.1$ (1 arom. C); $167.1\left(\mathrm{CO}_{2}\right) ; 181.9(\mathrm{C}(2)) ; 193.2$ (CO). CI-MS: 314 (20), 313 (15), 312 (100, [M+1] $\left.]^{+}\right), 311$ (8, $\left.\mathrm{M}^{+}\right), 310$ (52), 309 (19), 308 (19). Anal. Calcd for $\mathrm{C}_{13} \mathrm{H}_{13} \mathrm{NO}_{3} \mathrm{Se}$ (311.01): C, 50.33; H, 4.22; N, 4.52. Found: C, 50.43; H, 4.38; N, 4.50.

\section{Ethyl 4,5-dihydro-2-[(4-methylphenyl)amino]-4-oxoselenophene-3-carboxylate (4b)}

Yield: 56\%. White crystals. M.p. $151-153^{\circ} \mathrm{C}$. IR (KBr): $3425 w$ br, 3224w, 1662s, $1636 s, 1565 s$, $1513 m, 1470 w, 1416 m, 1374 m, 1224 s, 1190 w, 1043 m, 780 w .{ }^{1} \mathrm{H}^{\mathrm{NMR}}\left(\mathrm{CDCl}_{3}\right): 1.55$ (t, J=7.2 Hz, 3H, $\left.\mathrm{CH}_{3}\right) ; 2.55$ (s, 3H, $\left.\mathrm{CH}_{3}\right) ; 3.85$ (s, 2H, $\left.\mathrm{CH}_{2}\right) ; 4.52$ (q, J=7.1 Hz, 2H, $\left.\mathrm{CH}_{2} \mathrm{O}\right) ; 7.40$ (s, 4H, arom. CH). ${ }^{13} \mathrm{C}$ NMR $\left(\mathrm{CDCl}_{3}\right)$ : $14.3\left(\mathrm{CH}_{3}\right) ; 21.0\left(\mathrm{CH}_{3}\right) ; 32.6\left(\mathrm{CH}_{2}\right) ; 60.4\left(\mathrm{CH}_{2} \mathrm{O}\right) ; 99.6(\mathrm{C}(3)) ; 124.2(2$ arom. $\mathrm{CH})$; 130.2 (2 arom. CH); 135.6 (1 arom. C); 138.2 (1 arom. C); $167.1\left(\mathrm{CO}_{2}\right) ; 182.2$ (C(2)); 193.2 (CO). CIMS: 328 (18), $327(9), 326\left(100,[\mathrm{M}+1]^{+}\right), 325\left(19, \mathrm{M}^{+}\right), 324(50), 323$ (23), 322 (22). Anal. Calcd for $\mathrm{C}_{14} \mathrm{H}_{15} \mathrm{NO}_{3} \mathrm{Se}$ (325.02): C, 51.86; H, 4.66; N, 4.32. Found: C, 51.95; H, 4.83; N, 4.31.

Suitable crystals for the X-ray crystal-structure determination were grown from $\mathrm{CH}_{2} \mathrm{Cl}_{2}$ by slow evaporation of the solvent.

\section{Ethyl 2-[(4-bromophenyl)amino]-4,5-dihydro-4-oxoselenophene-3-carboxylate (4c)}

Yield: $54 \%$. Yellowish crystals. M.p. $186-188^{\circ} \mathrm{C}$. IR (KBr): $3442 w$ br, 3142w, 1658s, 1640s, $1555 s, 1529 m, 1489 w, 1410 m, 1377 m, 1349 w, 1269 w, 1217 m, 1066 w, 1035 w, 1011 w, 782 w .{ }^{1} \mathrm{H}$ NMR $\left(\mathrm{CDCl}_{3}\right): 1.33\left(\mathrm{t}, \mathrm{J}=7.2 \mathrm{~Hz}, 3 \mathrm{H}, \mathrm{CH}_{3}\right) ; 3.65\left(\mathrm{~s}, 2 \mathrm{H}, \mathrm{CH}_{2}\right) ; 4.30\left(\mathrm{q}, \mathrm{J}=7.1 \mathrm{~Hz}, 2 \mathrm{H}, \mathrm{CH}_{2} \mathrm{O}\right) ; 7.19$ (d, J=8.2 
$\mathrm{Hz}, 2 \mathrm{H}$, arom. $\mathrm{CH}) ; 7.52(\mathrm{~d}, \mathrm{~J}=8.2 \mathrm{~Hz}, 2 \mathrm{H}$, arom. $\mathrm{CH}) .{ }^{13} \mathrm{C} \mathrm{NMR}\left(\mathrm{CDCl}_{3}\right): 14.3\left(\mathrm{CH}_{3}\right) ; 32.8\left(\mathrm{CH}_{2}\right) ; 60.5$ $\left(\mathrm{CH}_{2} \mathrm{O}\right) ; 100.1$ (C(3)); 121.6 (1 arom. C); 125.8 (2 arom. $\left.\mathrm{CH}\right) ; 132.9$ (2 arom. $\left.\mathrm{CH}\right) ; 137.1$ (1 arom. C); $167.2\left(\mathrm{CO}_{2}\right)$; $182.1(\mathrm{C}(2))$; $193.0(\mathrm{CO})$. CI-MS: 392 (78), 391 (34), $390\left(100,[\mathrm{M}+1]^{+}\right), 389\left(23, \mathrm{M}^{+}\right)$, 388 (47), 387 (19). Anal. Calcd for $\mathrm{C}_{13} \mathrm{H}_{12} \mathrm{NO}_{3} \mathrm{SeBr}$ (388.92): C, 40.13; H, 3.11; N, 3.60. Found: C, $40.31 ; \mathrm{H}, 3.24 ; \mathrm{N}, 3.56$.

\section{Ethyl 4,5-dihydro-2-[(2,6-dimethylphenyl)amino]-4-oxoselenophene-3-carboxylate (4d)}

Yield: $26 \%$. White crystals. M.p. $163-165^{\circ} \mathrm{C}$. IR (KBr): $3441 w b r, 3202 w, 2979 w, 1661 s, 1641 s$, $1593 w, 1549 s, 1474 w, 1404 m, 1378 w, 1352 w, 1273 w, 1219 m, 1029 m, 790 w .{ }^{1} \mathrm{H} \mathrm{NMR}\left(\mathrm{CDCl}_{3}\right): 1.33(\mathrm{t}$, $\left.\mathrm{J}=7.2 \mathrm{~Hz}, 3 \mathrm{H}, \mathrm{CH}_{3}\right) ; 2.21\left(\mathrm{~s}, 6 \mathrm{H}, 2 \mathrm{CH}_{3}\right) ; 3.59\left(\mathrm{~s}, 2 \mathrm{H}, \mathrm{CH}_{2}\right) ; 4.28\left(\mathrm{q}, \mathrm{J}=7.1 \mathrm{~Hz}, 2 \mathrm{H}, \mathrm{CH}_{2} \mathrm{O}\right) ; 7.07$ (d, $\mathrm{J}=8.2 \mathrm{~Hz}, 2 \mathrm{H}$, arom. $\mathrm{CH}) ; 7.20(\mathrm{t}, \mathrm{J}=8.2 \mathrm{~Hz}, 1 \mathrm{H}$, arom. $\mathrm{CH}) ; 10.98$ (br s, $1 \mathrm{H}, \mathrm{NH}) .{ }^{13} \mathrm{C} \mathrm{NMR}\left(\mathrm{CDCl}_{3}\right)$ : $14.3\left(\mathrm{CH}_{3}\right) ; 17.8\left(2 \mathrm{CH}_{3}\right) ; 32.4\left(\mathrm{CH}_{2}\right) ; 60.2\left(\mathrm{CH}_{2} \mathrm{O}\right)$; $99.0(\mathrm{C}(3))$; 128.7 (2 arom. $\left.\mathrm{CH}\right) ; 129.3$ (arom. $\left.\mathrm{CH}\right)$; 135.9 (1 arom. C); 136.1 (2 arom. C); $166.9\left(\mathrm{CO}_{2}\right) ; 185.2$ (C(2)); 193.2 (CO). CI-MS: 342 (19), 341 (8), $340\left(100,[\mathrm{M}+1]^{+}\right), 339\left(21, \mathrm{M}^{+}\right), 338$ (54), 337 (19), 336 (19). Anal. Calcd for $\mathrm{C}_{13} \mathrm{H}_{12} \mathrm{NO}_{3} \mathrm{SeBr}$ (339.04): C, 53.26; H, 5.07; N, 4.14. Found: C, 53.20; H, 5.27; N, 3.97.

\section{Ethyl 4,5-dihydro-2-[(4-methoxyphenyl)amino]-4-oxoselenophene-3-carboxylate (4e)}

Yield: 51\%. White crystals. M.p. $169-171^{\circ} \mathrm{C}$. IR (KBr): $3441 w \mathrm{br}, 1638 s, 1607 w, 1544 s, 1511 \mathrm{~m}$, $1461 w, 1385 w, 1336 w, 1240 w, 1212 m, 1183 w, 1028 m, 828 w, 786 w .{ }^{1} \mathrm{H}$ NMR $\left(\mathrm{CDCl}_{3}\right): 1.45(\mathrm{t}, \mathrm{J}=7.1$ $\left.\mathrm{Hz}, 3 \mathrm{H}, \mathrm{CH}_{3}\right) ; 3.72$ (s, 2H, $\left.\mathrm{CH}_{2}\right) ; 3.89$ (s, 3H, $\mathrm{CH}_{3} \mathrm{O}$ ); 4.41 (q, J=7.1 Hz, 2H, $\mathrm{CH}_{2} \mathrm{O}$ ); 7.01 (d, J=8.2 Hz, $2 \mathrm{H}$, arom. $\mathrm{CH}) ; 7.32(\mathrm{~d}, \mathrm{~J}=8.2 \mathrm{~Hz}, 2 \mathrm{H}$, arom. $\mathrm{CH}) ; 11.54$ (br s, $1 \mathrm{H}, \mathrm{NH}) .{ }^{13} \mathrm{C} \mathrm{NMR}\left(\mathrm{CDCl}_{3}\right): 14.3\left(\mathrm{CH}_{3}\right)$; $32.6\left(\mathrm{CH}_{2}\right) ; 55.4\left(\mathrm{CH}_{3} \mathrm{O}\right) ; 60.3\left(\mathrm{CH}_{2} \mathrm{O}\right) ; 99.4(\mathrm{C}(3)) ; 114.7$ (2 arom. $\left.\mathrm{CH}\right) ; 126.2$ (2 arom. $\left.\mathrm{CH}\right) ; 130.9$ (1 arom. C); 159.3 (1 arom. C); $167.1\left(\mathrm{CO}_{2}\right) ; 183.0$ (C(2)); 193.2 (CO). CI-MS: 344 (8), 343 (33), 342 $\left(100,[\mathrm{M}+1]^{+}\right), 341\left(12, \mathrm{M}^{+}\right), 340$ (42), 339 (10), 338 (9). Anal. Calcd for $\mathrm{C}_{14} \mathrm{H}_{15} \mathrm{NO}_{4} \mathrm{Se}$ (341.02): C, 49.42; H, 4.44; N, 4.12. Found: C, 49.66; H, 4.62; N, 4.02.

\section{Ethyl 2-cyclohexylamino-4,5-dihydro-4-oxoselenophene-3-carboxylate (4f)}

Yield: 44\%. Orange oil. IR (film): $3173 w$ br, 2933s, 2856m, 1729m br, 1642s br, 1561s, 1465m, $1451 m, 1408 s, 1382 m, 1350 m, 1304 m, 1268 m, 1218 m, 1149 w, 1094 w, 1032 m, 916 w, 891 w, 858 w$, 790m, 732m. ${ }^{1} \mathrm{H}$ NMR $\left(\mathrm{CDCl}_{3}\right)$ : 1.19-1.47 (m, 7H, $\left.\mathrm{CH}_{3}, 2 \mathrm{CH}_{2}\right) ; 1.95-1.99$ (m, 2H, $\left.\mathrm{CH}_{2}\right) ; 1.58-1.74(\mathrm{~m}$, $\left.4 \mathrm{H}, 2 \mathrm{CH}_{2}\right) ; 3.65\left(\mathrm{~s}, 2 \mathrm{H}, \mathrm{CH}_{2}\right) ; 3.26-3.31(\mathrm{~m}, 1 \mathrm{H}, \mathrm{CH}) ; 4.23\left(\mathrm{q}, \mathrm{J}=7.1 \mathrm{~Hz}, 2 \mathrm{H}, \mathrm{CH}_{2} \mathrm{O}\right) ; 10.11$ (br s, $1 \mathrm{H}$, $\mathrm{NH}) .{ }^{13} \mathrm{C} \mathrm{NMR}\left(\mathrm{CDCl}_{3}\right) \square 14.3\left(\mathrm{CH}_{3}\right) ; 24.1\left(2 \mathrm{CH}_{2}\right) ; 24.9\left(\mathrm{CH}_{2}\right) ; 32.5\left(2 \mathrm{CH}_{2}\right) ; 57.5(\mathrm{CH}) ; 60.0$ 
$\left(\mathrm{CH}_{2} \mathrm{O}\right) ; 87.0(\mathrm{C}(3)) ; 157.4(\mathrm{C}(2)) ; 167.3\left(\mathrm{CO}_{2}\right) ; 196.5(\mathrm{CO})$. CI-MS: 320 (11), 319 (34), 318 (100, $\left.[\mathrm{M}+1]^{+}\right), 317\left(8, \mathrm{M}^{+}\right), 316(44), 315$ (9), 314 (12). Anal. Calcd for $\mathrm{C}_{13} \mathrm{H}_{19} \mathrm{NO}_{3} \mathrm{Se}$ (317.05): C, 49.37; $\mathrm{H}$, 6.06; N, 4.43. Found: C, 49.46; H, 5.92; N, 4.22.

\section{Crystal-Structure Determination [27]}

A crystal of $\mathbf{4 b}$, obtained from $\mathrm{CH}_{2} \mathrm{Cl}_{2}$, was mounted on a glass fibre and used for a lowtemperature X-ray structure determination. All measurements were made on a Nonius KappaCCD areadetector diffractometer [28] using graphite-monochromated $\operatorname{Mo} K_{\alpha}$ radiation $(\lambda=0.71073 \AA)$ and an Oxford Cryosystems Cryostream 700 cooler. Data reduction was performed with HKL Denzo and Scalepack [29]. The intensities were corrected for Lorentz and polarization effects, and an absorption correction based on the multi-scan method [30] was applied. Equivalent reflections were merged. Data collection and refinement parameters are given in [31]. A view of the molecule is shown in Figure 1. The structure was solved by direct methods using SIR92 [32], which revealed the positions of all nonhydrogen atoms. The non-hydrogen atoms were refined anisotropically. The amine $\mathrm{H}$-atom was placed in the position indicated by a difference electron density map and its position was allowed to refine together with an isotropic displacement parameter. All remaining $\mathrm{H}$-atoms were placed in geometrically calculated positions and refined using a riding model where each $\mathrm{H}$-atom was assigned a fixed isotropic displacement parameter with a value equal to $1.2 \mathrm{U}_{\mathrm{eq}}$ of its parent $\mathrm{C}$-atom $(1.5 \mathrm{U}$ eq for the methyl groups). Refinement of the structure was carried out on $F^{2}$ using full-matrix least-squares procedures, which minimized the function $\Sigma w\left(F_{\mathrm{o}}{ }^{2}-F_{\mathrm{c}}{ }^{2}\right)^{2}$. A correction for secondary extinction was applied. Neutral atom scattering factors for non-hydrogen atoms were taken from [33a], and the scattering factors for $\mathrm{H}$ atoms were taken from [34]. Anomalous dispersion effects were included in $F_{\mathrm{c}}$ [35]; the values for $f^{\prime}$ and $f^{\prime \prime}$ were those of [33b]. The values of the mass attenuation coefficients are those of [33c]. All calculations were performed using the SHELXL97 [36] program.

\section{ACKNOWLEDGMENTS}

We thank the analytical units of our institute for spectra and analyses. Financial support of this work by the Dr. Helmut Legerlotz Foundation (postdoctoral stay of G.L.S. at the University of Zürich, 8.2004 8.2005) and F. Hoffmann-La Roche AG, Basel, is gratefully acknowledged. 


\section{REFERENCES}

[1] Koketsu, M.; Ishihara, H. Curr. Org. Chem., 2003, 7, 175.

[2] (a) Sommen, G. L. Mini Rev. Org. Chem. 2005, 2, 375; (b) Shatz, J. Science of Synthesis: Product Class 11: Selenophenes, 2002, 9, 423; (c) Magdesevia, N. N. Adv. Heterocycl. Chem., 1970, 12, 1; (d) Hörnfeldt, A.-B. Adv. Heterocycl. Chem., 1982, 30, 127.

[3] (a) Sommen, G. L.; Comel, A.; Kirsch, G. Synlett, 2001, 11, 1731; (b) Sommen, G. L.; Comel, A.; Kirsch, G. Tetrahedron Lett., 2002, 1, 237; (c) Augustin, M.; Rudorf, W. D.; Schmidt, U. Tetrahedron, 1976, 32, 3055; (d) Rudorf, W. D.; Schierhorn, A.; Augustin, M. Tetrahedron, 1979, 35, 551.

[4] (a) Faull, A. W.; Hull, R. J. Chem. Soc., Perkin Trans. I, 1981, 1078; (b) Faull, A. W.; Hull, R. J. Chem. Res.(S), 1979, 240.

[5] Ibrahim, N. S.; Sadek, K. U.; Aziz, S. I.; Elnagdi, M. H. Z. Naturforsch., 1985, 40B, 129.

[6] Mack, R. A.; Zazulak, W. I.; Radov, L. A.; Baer, J. E.; Stewart, J. D.; Elzer, P. H.; Kinsolving, C. R.; Georgiev, V. S. J. Med. Chem., 1988, 31, 1910.

[7] Atanassov, P. K.; Linden, A.; Heimgartner, H. Helv. Chim. Acta, 2004, 87, 1873.

[8] Atanassov, P. K.; Linden, A.; Heimgartner, H. Helv. Chim. Acta, 2004, 87, 1452.

[9] Atanassov, P. K.; Linden, A.; Heimgartner, H. Heterocycles, 2004, 62, 521.

[10] Atanassov, P. K.; Linden, A.; Heimgartner, H. Heterocycles, 2003, 61, 569.

[11] Atanassov, P. K.; Linden, A.; Heimgartner, H. Helv. Chim. Acta, 2003, 86, 3235.

[12] Atanassov, P. K.; Zhou, Y.; Linden, A.; Heimgartner, H. Helv. Chim. Acta 2002, 85, 1102.

[13] Zhou, Y.; Heimgartner, H. Helv. Chim. Acta, 2000, 83, 539.

[14] Zhou, Y.; Linden, A.; Heimgartner, H. Helv. Chim. Acta, 2000, 83, 1576.

[15] Sommen, G. L.; Linden, A.; Heimgartner, H. Helv. Chim. Acta, 2005, 88, 766.

[16] Sommen, G. L.; Linden, A.; Heimgartner, H. Eur. J. Org. Chem., 2005, 3128.

[17] Sommen, G. L.; Linden, A.; Heimgartner, H. Tetrahedron, 2006, 62, 3344.

[18] Sommen, G. L.; Linden, A.; Heimgartner, H. Helv. Chim. Acta, accepted.

[19] Sommen, G. L.; Linden, A.; Heimgartner, H. Helv. Chim. Acta, 2006, 89, 1322.

[20] Sommen, G. L.; Linden, A.; Heimgartner, H. Tetrahedron Lett., 2005, 46, 6723.

[21] Sommen, G. L.; Linden, A.; Heimgartner, H. Heterocycles, 2005, 65, 1903.

[22] Favero, F.; Sommen, G. L.; Linden, A.; Heimgartner, H. Heterocycles, 2006, 67, 749. 
[23] (a) Barton, D. H. R.; Parekh, S. I.; Tajbakhsh, M.; Theodorakis, E. A.; Tse, C.-L. Tetrahedron, 1994, 50, 639; (b) Bakhsh, M. T.; Behshtiha, Y. S.; Heravi, M. M. J. Chem. Soc. Pakistan, 1996, $18,159$.

[24] Johnson, C. K. ORTEP II, Report ORNL-5138, Oak Ridge National Laboratory, Oak Ridge, Tennessee, 1976.

[25] Bernstein, J.; Davis, R. E.; Shimoni, L.; Chang, N.-L. Angew. Chem. Int. Ed. Engl., 1995, 34, 1555.

[26] Katritzky, A. R.; Parris, R. L.; Ignatchenko, E. S.; Allin, S. M.; Siskin M. J. Prakt. Chem. 1997, $339,59$.

[27] CCDC-629496 contains the supplementary crystallographic data for this paper. These data can be obtained free of charge from the Cambridge Crystallographic Data Centre CCDC via http://www.ccdc.ac.uk/data_request/cif.

[28] Hooft, R. KappaCCD Collect Software, Nonius BV, Delft, The Netherlands, 1999.

[29] Otwinowski, Z., Minor, W. in Methods in Enzymology, Vol. 276, Macromolecular Crystallography, Part A, Eds. Carter, C. W. Jr.; Sweet, R. M., Academic Press, New York, 1997, 307.

[30] Blessing, R.H. Acta Crystallogr., Sect A 1995, 51, 33.

[31] Crystallographic data for compound $4 \mathbf{b}$ : Empirical formula: $\mathrm{C}_{14} \mathrm{H}_{15} \mathrm{NO}_{3} \mathrm{Se}$; formula weight: $324.18 \mathrm{~g} \mathrm{~mol}^{-1}$; crystal color, habit: colorless, prism; crystal dimensions: $0.20 \times 0.30 \times 0.30 \mathrm{~mm}$; temperature: $160(1) \mathrm{K}$; crystal system: monoclinic; space group: $C 2 / c ; Z=8$; reflections for cell determination: 28982; $2 \theta$ range for cell determination: $4-60^{\circ}$; unit cell parameters: $a=$ 26.8226(4) $\AA, b=7.2506(1) \AA, c=14.1780(2) \AA, \beta=97.811(1)^{\circ}, V=2731.76(7) \AA^{3}, D_{x}=$ $1.576 \mathrm{~g} \mathrm{~cm}^{-3} ; \mu\left(\mathrm{MoK}_{\alpha}\right)=2.750 \mathrm{~mm}^{-1}$; scan type: $\phi$ and $\omega ; 2 \theta_{(\max )}=60^{\circ}$; transmission factors (min; $\max$ ): 0.483 ; 0.581 ; total reflections measured: 41421 ; symmetry independent reflections: 3990; reflections with $I>2 \sigma(I)$ : 3431; reflections used in refinement: 3990; parameters refined: 179; final $R(F)\left[I>2 \sigma(I)\right.$ reflections]: 0.0319; $w R\left(F^{2}\right)$ (all data):0.0820; weights: $w=\left[\sigma^{2}\left(F_{\mathrm{o}}^{2}\right)\right.$ $\left.+(0.0331 P)^{2}+3.6031 P\right]^{-1}$ where $P=\left(F_{\mathrm{o}}^{2}+2 F_{\mathrm{c}}^{2}\right) / 3$; goodness of fit: 1.136 ; secondary extinction coefficient: $0.0012(2)$; final $\Delta_{\max } / \sigma=0.001 ; \Delta \rho(\max ; \min )=0.46 ;-0.60\left[\mathrm{e} \AA^{-3}\right]$.

[32] Altomare, A.; Cascarano, G.; Giacovazzo, C.; Guagliardi, A.; Burla, M. C.; Polidori, G.; Camalli, M. SIR92, J. Appl. Crystallogr. 1994, 27, 435. 
[33] (a) Maslen, E. N.; Fox, A. G.; O'Keefe, M. A. in 'International Tables for Crystallography', Ed. Wilson, A. J. C., Kluwer Academic Publishers, Dordrecht, 1992, Vol. C, Table 6.1.1.1, p. 477; b) Creagh, D. C.; McAuley, W. J. ibid. Table 4.2.6.8, p. 219; c) Creagh, D. C.; Hubbell, J. H. ibid.4] Stewart, R. F.; Davidson, E. R.; Simpson, W. T. J. Chem. Phys. 1965, 42, 3175.

[35] Ibers, J. A.; Hamilton, W. C. Acta Crystallogr. 1964, 17, 781.

[36] Sheldrick, G. M. SHELXL97, Program for the Refinement of Crystal Structures, University of Göttingen, Germany, 1997. 